\title{
Fluctuations and the rapidity dependence of charged particles spectra in fixed centrality bins in $\mathrm{p} A$ collisions
}

\author{
Larry McLerran ${ }^{\mathrm{a}, \mathrm{b}}$, Michal Praszalowicz ${ }^{\mathrm{c}}$ \\ ${ }^{a}$ Physics Dept, Bdg. 510A, Brookhaven National Laboratory, Upton, NY-11973, USA \\ ${ }^{b}$ Physics Dept, China Central Normal University, Wuhan, China \\ ${ }^{c}$ M. Smoluchowski Institute of Physics, Jagiellonian University, \\ ul. S. Eojasiewicza 11, 30-348 Kraków, Poland.
}

\begin{abstract}
We argue that large fluctuations in the saturation momentum are necessary to explain the ATLAS and ALICE data on $\mathrm{p} A$ collisions measured at the LHC. Using a form for the distribution of fluctuations motivated by theoretical studies of the non-linear evolution equations for the Color Glass Condensate, we find a remarkably good agreement between theory and the measured distributions. If the saturation momentum fluctuates, we argue that the cross section for a proton probe should also fluctuate, consistent with previous observations.
\end{abstract}

\section{Introduction}

In a recent paper by Bzdak and Skokov [1], it was argued that the number of participants dependence of the multiplicity as a function of centrality provided a crucial test which might discriminate between the description of heavy ion collisions provided by the Color Glass Condensate[2] and that of the wounded nucleon model[3,4]. Their basic idea is very simple: In the simplest version of the wounded nucleon model, the rapidity dependence of the multiplicity distribution for a collision of a projectile nucleon against $N_{\text {part }}-1$ nucleons in the target is a simple triangular distribution going from a number proportional the number or participants for rapidities close to that of the nucleus to a number of order 1 at rapidities corresponding to the beam nucleon. This is shown in Fig. 1 


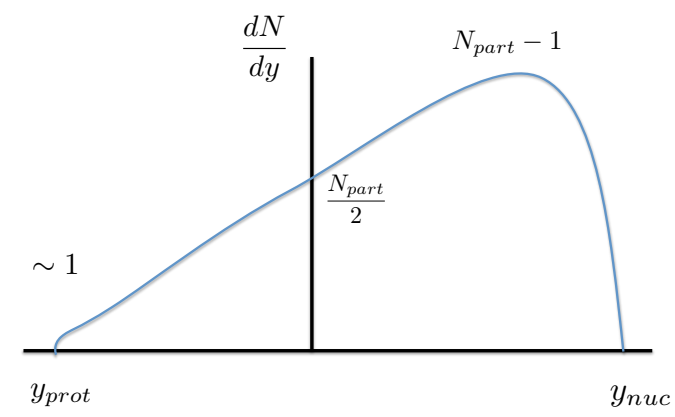

Figure 1: An illustration of the multiplicity distribution of produced particles in $\mathrm{p} A$ collisions as a function of rapidity in the simplest version of the wounded nucleon model.

On the other hand, the simplest version of the Color Glass Condensate model is that the rapidity distribution in the fragmentation of the target nucleus is of order $N_{\text {part }}$ but elsewhere of order 1 up to logarithms of the number of participants. This is because for a probe which has a Lorentz gamma factor of order $\gamma \geq A^{1 / 3}$ relative to the nucleus, the nucleons in the nucleus act coherently, and for the typical transverse momentum scale associated with particle production, the nucleus appears as a black disk. The multiplicity is controlled by the number of partons in the proton evolved to the saturation scale of the nucleus. In fact there is a mild logarithmic correction to this, so the multiplicity scales as $\ln N_{\text {part }}[5,6]$. Schematically the Color Glass Condensate description is shown in Fig. 2.

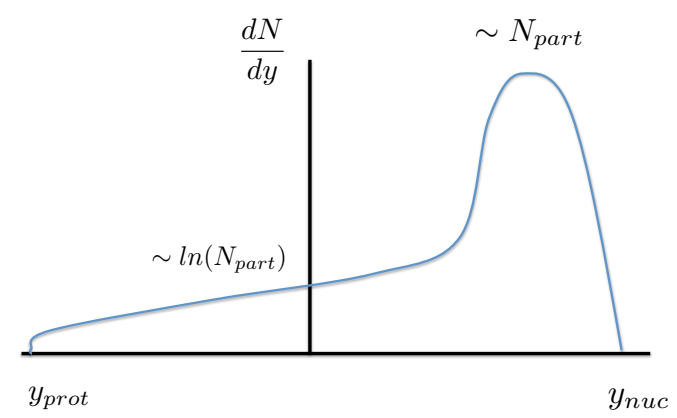

Figure 2: An illustration of the multiplicity distribution of produced particles in $\mathrm{p} A$ collisions as a function of rapidity in the simplest version of a model for the Color Glass Condensate.

The number of participants dependence of the pseudorapidity distributions in $\mathrm{p} A$ collisions at the LHC energy has been recently measured by the 
ATLAS [7] and ALICE [8] collaborations. In ATLAS, they extract the number of participant dependence of the integrated multiplicity under a variety of different assumptions concerning the cross-section for the proton penetrating through the nucleus. These correspond to a fixed Glauber cross section for the proton and to two different parameterizations of the Glauber-Gribov description $[9,10]$. The Glauber-Gribov model allows the proton cross-section to vary by Gausssian fluctuations. Similar procedures have been also applied by the ALICE experiment.

In the analysis of the next section, we compare expectations from the naive versions of the wounded nucleon model and that of the Color Glass Condensate to the experimental data. This analysis consists of comparing the $N_{\text {part }}$ dependence predicted by the two models as a function of rapidity to that of the experimental data. Neither model gives an acceptable description of the data for any extraction of the number of participant dependence.

How might one resolve this impasse? A hint is suggested by the extraction of the relationship between number of participants and multiplicity used to determine centrality. One might argue that in the fragmentation region of the nucleus, all models agree that the multiplicity dependence is linear in the number of participants. If so, the closest we come to the fragmentation region with the data is for $2<\eta<2.7$. While this is not forward enough to really be in the nuclear fragmentation region, we see that the data favors large fluctuations in the proton cross section. This suggests there might be fluctuations in the proton saturation momentum.

Before we discuss fluctuations of the saturation scale, let us remark that there exists a number of theoretical papers that took an effort to describe the $\mathrm{p} A$ data within the framework of the different versions of the CGC-based models. A complete list of such models can be found in a review paper [11] that was, however, published before the first LHC run. More recently successful description of the $\mathrm{p} A$ data has been presented in Refs. [12, 13, 14, $15]$ and references therein.

One expects a relationship between the cross section and the saturation momentum. If we require that the local density of gluons, denoted as $\Lambda^{2}$, in an impact parameter model of the proton factorizes as proportional to the number of gluons per unit area on average times an impact parameter profile, which for simplicity can be assumed to be exponential, then

$$
\Lambda^{2}=Q_{\mathrm{s}}^{2} e^{-\mu R}
$$


Requiring that the cross-section corresponds to the radius at which this density has some fixed value $\Lambda_{0}^{2}$, perhaps $\Lambda_{\mathrm{QCD}}^{2}$, we conclude that the radius of the proton grows as

$$
R \sim \frac{1}{\mu} \ln \left(Q_{\mathrm{s}}^{2} / \Lambda_{0}^{2}\right)
$$

Therefore the cross section $\sigma \propto \ln ^{2}\left(Q_{\mathrm{s}}^{2} / \Lambda^{2}\right)$ will fluctuate if the saturation momentum does. There are of course many weaknesses of this analysis, perhaps the most important one is that we do not really know the impact parameter distribution for matter inside the proton, so that the exponential form, while valid at very large radii, may be misleading.

The emergence of the saturation scale and geometrical scaling in the theory of the Color Glass Condensate has been introduced in Refs. [16, 17]. It is based on the fact that the nonlinear evolution equations in small Bjorken $x$ regime $[18,19]$ possess traveling wave solutions $[16,17]$. It has been argued, however, in Ref. [23] and expanded in Ref. [24] that the scaling CGC solutions have exponential tails which are unphysical, and therefore have to be modified. This modification is based on the analogy with the statistical physics, and has clear interpretation in the dipole model, where the small tail of the amplitude is essentially counting a number of interacting dipoles. This discrete nature of the tail introduces stochastic diffusion that affects the shape of the entire amplitude, which - event by event - satisfies geometrical scaling, with the saturation scale that is, however, somewhat different than the one obtained in the mean field approximation. It has been argued that these fluctuations are Gaussian in the logarithm of the saturation momentum [23], and controlled by a probability distribution:

$$
\begin{aligned}
P(\rho) & =\frac{1}{\sqrt{2 \pi} \sigma} \exp \left(-\frac{\left(\ln Q_{\mathrm{s}}^{2} / Q_{0}^{2}-\ln Q_{p}^{2} / Q_{0}^{2}\right)^{2}}{2 \sigma^{2}}\right) \\
& =\frac{1}{\sqrt{2 \pi} \sigma} \exp \left(-\frac{\rho^{2}}{2 \sigma^{2}}\right)
\end{aligned}
$$

where

$$
\rho=\ln \frac{Q_{\mathrm{s}}^{2}}{Q_{p}^{2}} .
$$

Here $Q_{\mathrm{s}}^{2}$ is the proton saturation momentum fluctuating around its logarithmic average denoted as $\ln Q_{p}^{2}$ (with $Q_{0}^{2} \sim 1 \mathrm{GeV}^{2} / c^{2}$ being an arbitrary momentum scale, which cancels out in $(3))$. 
It has been furthermore argued in [23] that the width $\sigma$ of the distribution (3) is growing with the energy of the collision and depends upon the rapidity where measurements are made. One has to stress, however, that this result is only asymptotic, and - as the authors of Ref. [23] admit explicitly - there is no general analytical proof of this result and its status is still that of a conjecture. Phenomenologically scaling violations corresponding ro

$$
\sigma \sim \sqrt{y} \text { with } y=\log (1 / x)
$$

have been searched for in the DIS data with negative result [20, 21, 22].

There are two possible ways out from this situation. Either (5) holds down to the present energies (or presently accessible Bjorken $x$ 's), in which case the distribution (3) collapses to $\delta\left(\ln Q_{\mathrm{s}}^{2} / Q_{p}^{2}\right)$, or result (5) is only asymptotic, and for low energies $\sigma$ tends to a constant. As we shall show in the following, in such a case geometrical scaling would still hold.

To this end let us consider a DIS amplitude $T$ that exhibits geometrical scaling with respect to the fluctuating saturation momentum $Q_{\mathrm{s}}(x)$ :

$$
T\left(Q^{2} / Q_{s}^{2}\right)=T\left(\rho_{Q}-\rho\right)
$$

where $\rho_{Q}=\ln \left(Q^{2} / Q_{0}^{2}\right)$. Note that $T$ depends on $y=\ln (1 / x)$ through $x$ dependence of $Q_{\mathrm{s}}$. For illustrative purposes let's assume, following Ref. [24], that

$$
T\left(\rho_{Q}-\rho\right)=\Theta\left(\rho-\rho_{Q}\right)
$$

representing a step-like front moving with rapdity $y=\ln (1 / x)$, which plays a role of an evolution time. Averaging (7) with probability distribution (3) gives:

$$
\begin{aligned}
\left\langle T_{x}\left(\rho_{Q}\right)\right\rangle & \left.=\int_{-\infty}^{\infty} d \rho P(\rho) T\left(\rho_{Q}-\rho\right)\right) \\
& =\frac{1}{2} \operatorname{Erfc}\left(\frac{\log \left(Q^{2} / Q_{p}^{2}(x)\right)}{\sqrt{2} \sigma}\right) .
\end{aligned}
$$

So the new scaling variable is

$$
\log \tau=\frac{1}{\sigma} \log \left(Q^{2} / Q_{p}^{2}(x)\right)
$$


It is also straightforward to check that if $T$ is a function of $\rho_{Q}-\rho$ then, after integrating over fluctuations, $T \rightarrow\left\langle T\left(\rho_{p}-\rho_{Q}\right)\right\rangle$ so that geometrical scaling is preserved so long as the width of fluctuations is rapidity independent.

We do not have any explicit model leading to the fluctuations with the constant width, although - given the phenomenological arguments above such fluctuations are compatible with the DIS data. The fluctuations of the saturation momentum we are employing here are caused by an evolution with energy of the initial conditions of the forward scattering amplitude and are associated with the low density region (i.e. with a region where the amplitude is small, which means rather large Bjorken $x$ 's). But for large $x$ 's parton distributions are non-perturbative, and the fluctuations can be of order one. In contrast, it was established that pomeron loops effects [25], once at small $x$, are small, and therefore they cause only a small change in the scale of rapidity fluctuations as one goes to higher and higher energies. Large nonperturbative fluctuations in turn, propagate to small $x$ 's i.e. to the region we are interested in.

In the following shall ignore the rapidity dependence and treat $\sigma$ as a constant at fixed LHC energy. Obviously one needs to include beam energy dependence if this result would be used over a very wide range of energy from RHIC to the LHC.

In the analysis of $\mathrm{p} A$ collisions which we present below, we will consider fluctuations only of the nucleon. Including the fluctuations of the saturation scale in the nucleus might be done, but we expect a small effect. This is because the fractional change in the saturation momentum squared of the nucleus should be reduced by random walks. We might expect $\delta Q_{\mathrm{s}}^{2} / Q_{\mathrm{s}}^{2} \sim 1 / \sqrt{N_{A \text { part }}}$ where $N_{A \text { part }}$ is the number of participating nucleons in the target nucleus. In pp collisions, we would need to consider fluctuations in both protons. Without such inclusion of the effects of fluctuations of the nuclear saturation momentum, the results we present here should be taken to demonstrate qualitatively and semi-quantitatively the effects of fluctuating saturation momenta, and quantitative conclusions should await a more systematic analysis.

\section{Limitations of the Wounded Nucleon and Color Glass Descrip- tions of the $\mathrm{LHC} \mathrm{pPb}$ Data}

In the wounded nucleon (WN) model $[3,4]$ multiplicity is given in terms of the number of wounded nucleons multiplied by a function describing radiation 
of particles by one struck participant:

$$
\frac{d N}{d \eta}=w_{p} F_{p}(\eta)+w_{A} F_{A}(\eta)
$$

Total number of participants is therefore $N_{\text {part }}=w_{p}+w_{A}=1+w_{A}$. The only difference between functions $F_{p}$ and $F_{A}$ is that they radiate particles into different hemispheres. Therefore

$$
F_{p}(\eta)=F(\eta), F_{A}(\eta)=F(-\eta) .
$$

In order to test WN model against data it is convenient to introduce symmetrized and antisymmetrized spectra [4]:

$$
\begin{aligned}
& \left.\frac{d N}{d \eta}\right|_{\mathrm{sym}}=\frac{d N}{d y}(\eta)+\frac{d N}{d y}(-\eta)=\left(w_{p}+w_{A}\right)(F(\eta)+F(-\eta)), \\
& \left.\frac{d N}{d \eta}\right|_{\mathrm{asym}}=\frac{d N}{d \eta}(\eta)-\frac{d N}{d \eta}(-\eta)=\left(w_{p}-w_{A}\right)(F(\eta)-F(-\eta)) .
\end{aligned}
$$

Equation (12) implies that ratios of symmetrized spectra at different centralities are independent of $\eta$ and are equal to the ratio of number of participants. Ratios of symmetrized spectra to the centrality class with lowest number of participants are plotted in the left panel of Fig. 3. For clarity only in three cases error bands due to the uncertainties of $N_{\text {part }}$ are also displayed. We can see that an overall magnitude of these ratios agrees with the data, however - especially for large multiplicities - they are not $\eta$ independent in contrast to the prediction of the WN model.

On the other hand antisymmetrized spectra are proportional to $N_{\text {part }}-1$, assuming that on the proton side there was only one participant. We have fit the slope of the antisymmetrized spectra (which are to a good accuracy straight lines) to the highest $N_{\text {part }}$ data, obtaining predictions for other centralities. This is plotted in the right panel of Fig. 3 for three centrality classes. Shaded bands around the theoretical lines correspond again to the uncertainties of $N_{\text {part }}$. We see that here the wounded nucleon model fails to describe the data and that slopes of the antisymmetrized spectra have some additional dependence on $N_{\text {part }}$.

In the case of CGC, multiplicity is given in terms of the saturation scales[26]:

$$
\frac{d N}{d y}=S_{\perp} Q_{p}^{2}\left(2+\ln \frac{Q_{A}^{2}}{Q_{p}^{2}}\right)
$$



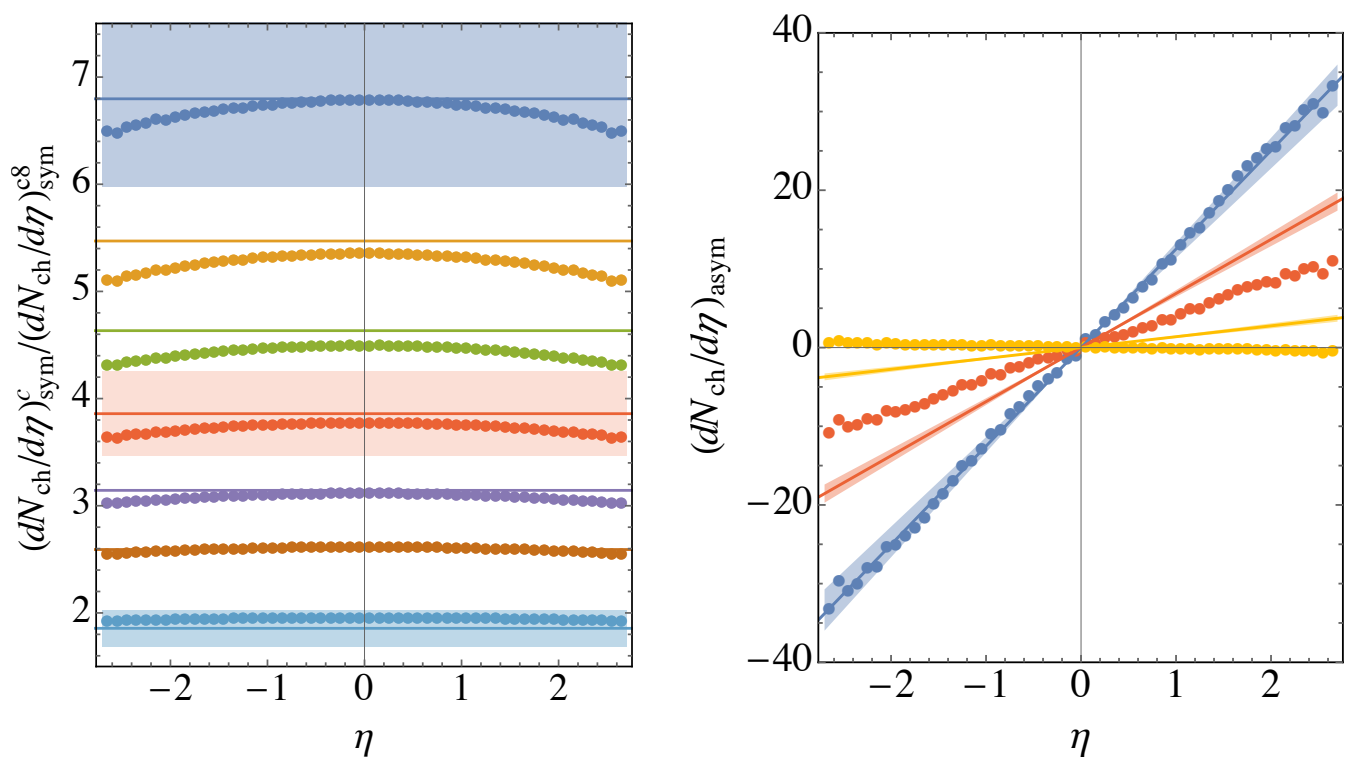

Figure 3: Wounded nucleon model predictions for symmetrized (left) and antisymmetrized (right) spectra defined in Eqs. (12) and (13). In the left panel ratios of symmetrized distributions to the 60-90\% (c8) centrality class are plotted together with the straight lines corresponding to the ratios of respective $N_{\text {part }}$. Error bands due to the uncertainty of $N_{\text {part }}$ are for clarity plotted only for three centrality classes: $0-1 \%$ (c1-top, blue), 10-20\% (c4 - middle, orange) and 40-50\% (c7 - bottom, light blue). In the right panel antisymmetrized spectra (13) are plotted for three centrality classes 0-1\% (blue), 10 $20 \%$ (orange) and 60-90\% (yellow). Shaded bands correspond to the uncertainty of $N_{\text {part }}$. Experimental points are from ATLAS Collaboration.

and the multiplicity in $\mathrm{p} A$ collisions is totally driven by the proton saturation scale. Here $S_{\perp}$ is a transverse area corresponding to an overlap of colliding hadrons. In the case of heavy ion and $\mathrm{p} A$ collisions it has rather well defined geometrical meaning, whereas in pp (small system) scattering it is a parameter related to the multiplicity of a given event. As such it may also fluctuate, however in the present paper such fluctuations are neglected. For fixed impact parameter

$$
\rho_{A}=\ln \frac{Q_{A}^{2}}{Q_{p}^{2}} \sim \ln N_{\text {part }}
$$

has only logarithmic dependence on $N_{\text {part }}$.

Formulae (14) and (15) predict both energy (denoted hereafter as $W=$ $\sqrt{s}$ ) dependence and rapidity (denoted below as $y$ ) dependence and also $N_{\text {part }}$ 
dependence of multiplicities through the dependence of the saturation scales on these quantities [26]:

$$
\begin{aligned}
& Q_{p}^{2}(W, y)=Q_{0}^{2}\left(\frac{W}{W_{0}}\right)^{\lambda} \exp (\lambda y) \\
& Q_{A}^{2}(W, y)=Q_{0}^{2} N_{\text {part }}\left(\frac{W}{W_{0}}\right)^{\lambda} \exp (-\lambda y)
\end{aligned}
$$

where we take for $\lambda=0.32$ in agreement with recent analysis [27] of the combined DIS data from HERA. Here $W_{0}$ is an arbitrary energy scale taking care of the proper dimension of the saturation scales.

Pseudo-rapidity $\eta$, which is measured, is related to rapidity $y$ in the following way

$$
y(\eta)=\frac{1}{2} \ln \left[\frac{\sqrt{\eta_{0}^{2}+\sinh ^{2} \eta}+\sinh \eta}{\sqrt{\eta_{0}^{2}+\sinh ^{2} \eta}-\sinh \eta}\right] .
$$

The corresponding Jacobian reads:

$$
h(\eta)=\frac{\cosh \eta}{\sqrt{\eta_{0}^{2}+\sinh ^{2} \eta}}
$$

where

$$
\eta_{0}^{2}=\frac{m^{2}+p_{\mathrm{T}}^{2}}{p_{\mathrm{T}}^{2}} .
$$

In the following we shall use $\eta_{0}^{2}=1.35$.

In Fig. 5 we plot theoretical predictions of Eq. (14) and the corresponding data from ATLAS and ALICE. One can see that CGC formula fails to describe overall normalization and the slope (note that theoretical curves have been normalized at $\eta=0$ ). In the remainder of this paper we shall show that inclusion of the fluctuations of the proton saturation scale is going to improve upon both issues

\section{Fluctuations of the saturation scale}

In this Section we shall derive formulae for the multiplicity of gluons in the fluctuating case with probability distribution given by Eq. (3). For fluctuating saturation scale $Q^{2}<Q_{A}^{2}$ multiplicity takes the form given by Eq. (14) with $Q_{p}^{2}$ replaced by $Q^{2}$, and for $Q_{A}^{2}<Q^{2}$ we simply interchange 
$Q_{A} \leftrightarrow Q$. With this, we have ignored a possible slow dependence upon the saturation momentum of the proton that affects the transverse area. This is slowly varying, like a logarithm squared, according to the result in the introduction, but we will later need to include such an effect to properly normalize the pseudorapidity distribution that we compute.

Now we can compute the multiplicity as

$$
\frac{d N}{d y}=S_{\perp} Q_{p}^{2}\left\{\int_{-\infty}^{\rho_{A}} d \rho\left(\rho_{A}-\rho+2\right) e^{\rho} P(\rho)+\int_{\rho_{A}}^{\infty} d \rho\left(\rho-\rho_{A}+2\right) e^{\rho_{A}} P(\rho)\right\}
$$

The first integral reads

$$
\begin{aligned}
I_{1} & =\int_{-\infty}^{\rho_{A}} d \rho\left(\rho_{A}-\rho+2\right) e^{\rho} P(\rho) \\
& =\frac{e^{\sigma^{2} / 2}}{\sqrt{2 \pi}}\left\{\sigma e^{-\left(\rho_{A}-\sigma^{2}\right)^{2} / 2 \sigma^{2}}+\sqrt{\frac{\pi}{2}}\left(\rho_{A}-\sigma^{2}+2\right)\left(1+\operatorname{Erf}\left(\frac{\rho_{A}-\sigma^{2}}{\sqrt{2} \sigma}\right)\right\}\right.
\end{aligned}
$$

and the remaining integral is

$$
\begin{aligned}
I_{2} & =\int_{\rho_{A}}^{\infty} d \rho\left(\rho-\rho_{A}+2\right) e^{\rho_{A}} P(\rho) \\
& =\frac{e^{\rho_{A}}}{\sqrt{2 \pi}}\left\{\sigma e^{-\rho_{A}^{2} / 2 \sigma^{2}}+\sqrt{\frac{\pi}{2}}\left(2-\rho_{A}\right)\left(1-\operatorname{Erf}\left(\frac{\rho_{A}}{\sqrt{2} \sigma}\right)\right\} .\right.
\end{aligned}
$$

This gives

$$
\frac{d N}{d y}=S_{\perp} Q_{p}^{2}\left(I_{1}+I_{2}\right)
$$

The saturation scales entering Eqs. (21)-(23) are given by Eq. (16); they depend on energy and rapidity, and - in the case of the nucleus - also on $N_{\text {part }}$. Note that in the limit where $\sigma \rightarrow 0$, i.e. in the limit with no fluctuations, $I_{2} \rightarrow 0$ and $I_{1} \rightarrow\left(2+\rho_{A}\right)$ in agreement with Eq. (14).

In order to illustrate the effect of fluctuations we plot in Fig. 4 the $\mathrm{p} A$ multiplicity for two different centrality classes for the non-fluctuating case (lower blue curve) and for the case with fluctuations included for $\sigma=1$ (middle orange line) and $\sigma=2$ (upper green line). We see clearly two effects whose strength depends on centrality: first, an overall normalization is increasing with increasing $\sigma$ and second, distributions are getting flatter when $\sigma$ is rising. For small number of participants we observe even a counterintuitive behavior that multiplicity is smaller on the nucleus side (upper green line in the left panel of Fig. 4). 

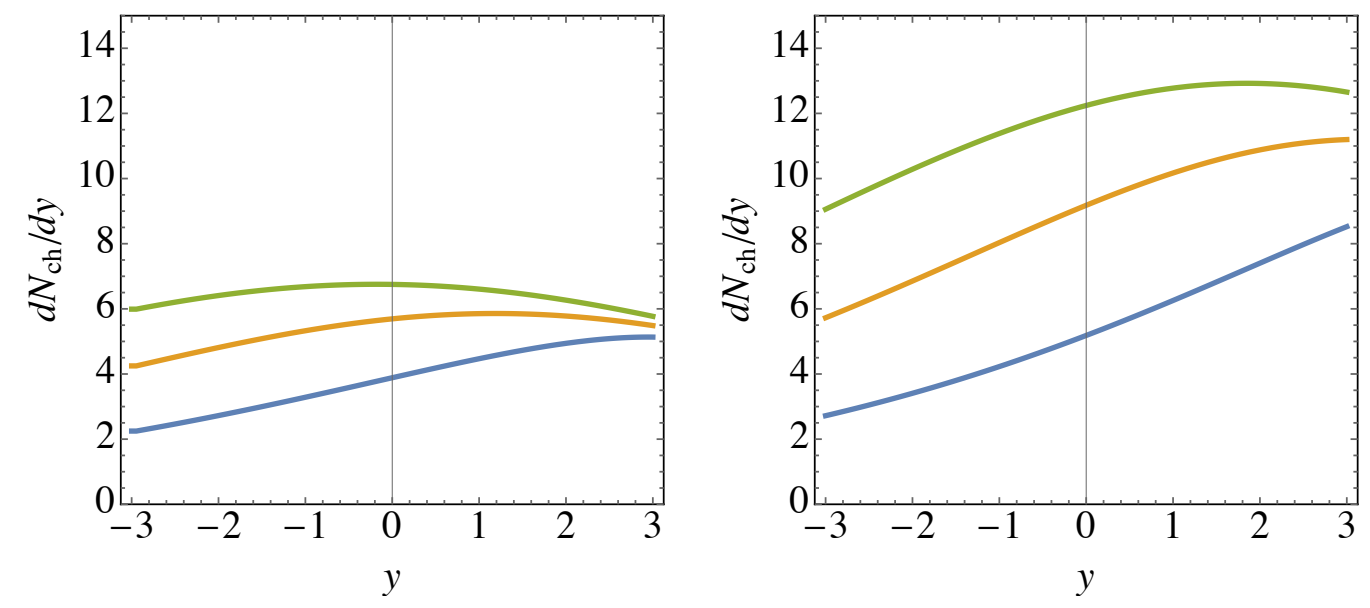

Figure 4: Multiplicity dependence on rapidity $y$ for the non-fluctating case (blue) and for the case with fluctuations with $\sigma=1$ (orange) and 2 (green). Left plot corresponds to $N_{\text {part }}=6.59$ and the right one to $N_{\text {part }}=24.13$.

\section{Multiplicity distributions in $\mathrm{p} A$}

Recently ATLAS and ALICE collaborations at the LHC have published multiplicity distributions in $\mathrm{pPb}$ collisions at $5.02 \mathrm{TeV}$ for different centrality classes summarized in Tables 1 and 2. Throughout this paper for ATLAS we have used $N_{\text {part }}$ determination by means of the fluctuating Glauber-Gribov model with $\Omega=0.55$ and for ALICE we use centrality classes determined by so called V0A method (for details see Refs. $[7,8]$ ). This data is shown in Figs. 5 and 6 . In Fig. 5 we also plot theoretical predictions for multiplicity distributions without fluctuations corresponding to Eq. (14). We see that CGC predictions are too steep. Moreover, they are not properly normalized, so we have adjusted the overall factor $S_{\perp}$ by normalizing the distributions at $\eta=0$. In this way $S_{\perp}$ does - as remarked in the Introduction - depend on $N_{\text {part }}$, and this dependence is plotted in Fig. 7.

Next in Fig. 6 we superimpose over the data theoretical predictions of Eqs. (21)-(23) with fluctuations included. Theoretical predictions are again normalized at $\eta=0$ and $S_{\perp}$ dependence on $N_{\text {part }}$ is plotted in Fig. 7 .

Before we discuss the effects of the fluctuations let us stress that we have tried to avoid playing with free parameters. Therefore we have kept $\lambda$ fixed to the DIS value of 0.32 , we have kept $\eta_{0}^{2}$ fixed to 1.35 . The only free parameter was $\sigma$ and normalization. We have found that the best description of both ATLAS and ALICE data is for $\sigma=1.55$. 


\begin{tabular}{|c|c|c|}
\hline class & $\%$ & $\left\langle N_{\text {part }}\right\rangle$ \\
\hline c1 & $0-1$ & 24.13 \\
c2 & $1-5$ & 19.42 \\
c3 & $5-10$ & 16.45 \\
c4 & $10-20$ & 13.70 \\
c5 & $20-30$ & 11.16 \\
c6 & $30-40$ & 9.20 \\
c7 & $40-60$ & 6.59 \\
c8 & $60-90$ & 3.55 \\
\hline
\end{tabular}

Table 1: Mean number of participants in different centrality classes as determined by ATLAS by Glauber-Gribov model with $\Omega=0.55$

\begin{tabular}{|c|c|c|}
\hline class & $\%$ & $\left\langle N_{\text {part }}\right\rangle$ \\
\hline c1 & $0-5$ & 15.7 \\
c2 & $5-10$ & 14.0 \\
c3 & $10-20$ & 12.7 \\
c4 & $20-40$ & 10.4 \\
c5 & $40-60$ & 7.42 \\
c6 & $60-80$ & 4.81 \\
c7 & $80-100$ & 2.94 \\
\hline
\end{tabular}

Table 2: Mean number of participants in different centrality classes as determined by ALICE by so called V0A method. 

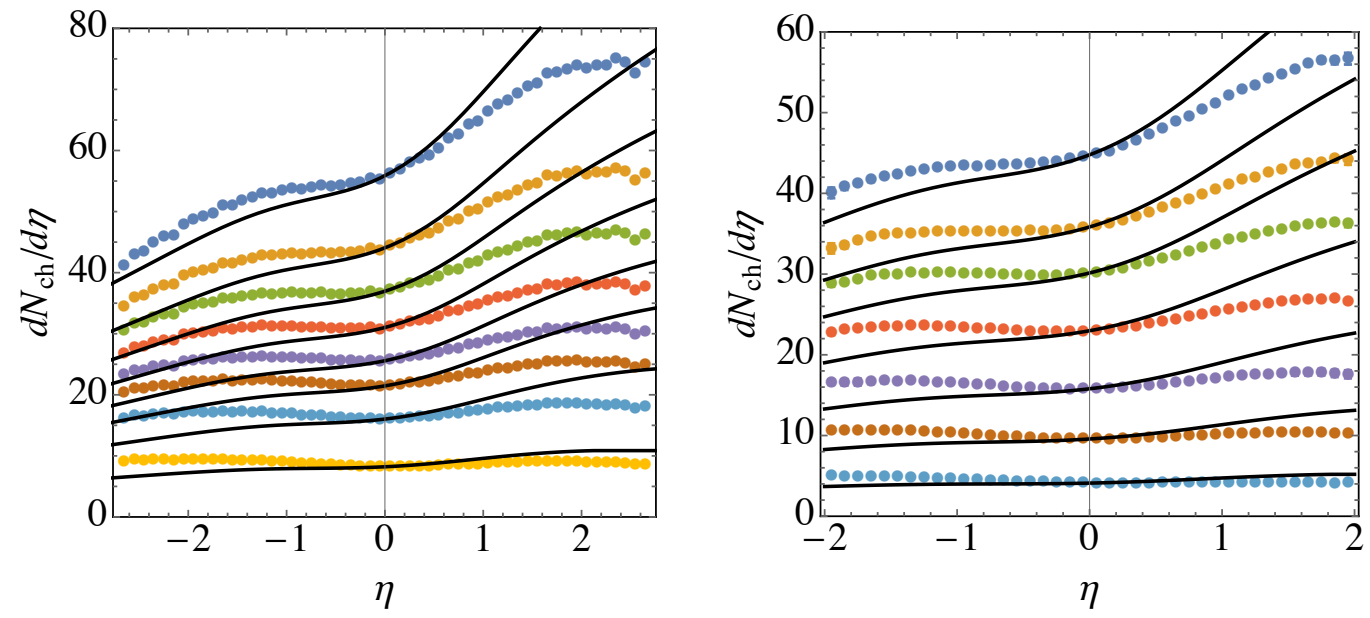

Figure 5: Multiplicity dependence on pseudorapidity $\eta$ given by formula (14) for ATLAS (left) and ALICE (right) for different centrality classes described in the text. Theoretical curves have been normalized at $\eta=0$ (see Fig. 7).

We see that fluctuations make $\eta$ distributions flatter and the $N_{\text {part }}$ dependence of $S_{\perp}$ is also weaker than in the case with no fluctuations. The dependence of $S_{\perp}$ can be well approximated by the following formulae:

$$
\begin{aligned}
& S_{\perp}^{\text {ATLAS }}=\left(0.66+0.6 \ln \left(N_{\text {part }}\right)\right)^{2}, \\
& S_{\perp}^{\text {ALICE }}=\left(0.09+0.84 \ln \left(N_{\text {part }}\right)\right)^{2}
\end{aligned}
$$

that take into account cross-section fluctuations with $N_{\text {part }}$ discussed in the Introduction. The strength of these fluctuations is, however, very different in the case of ATLAS and ALICE.

\section{Conclusions}

The original idea of studying multiplicity fluctuations in $\mathrm{p} A$ collisions was that they would allow probes of varying density regions inside the nucleus. The results we present in this paper suggest that the gluon content of the proton also itself changes when the multiplicity of final state particles varies. The analysis we present here attempts a semi-quantitative comparison to experimental data. This data is not simply explained by the simple applications of the theory of the Color Glass Condensate or of the Wounded 

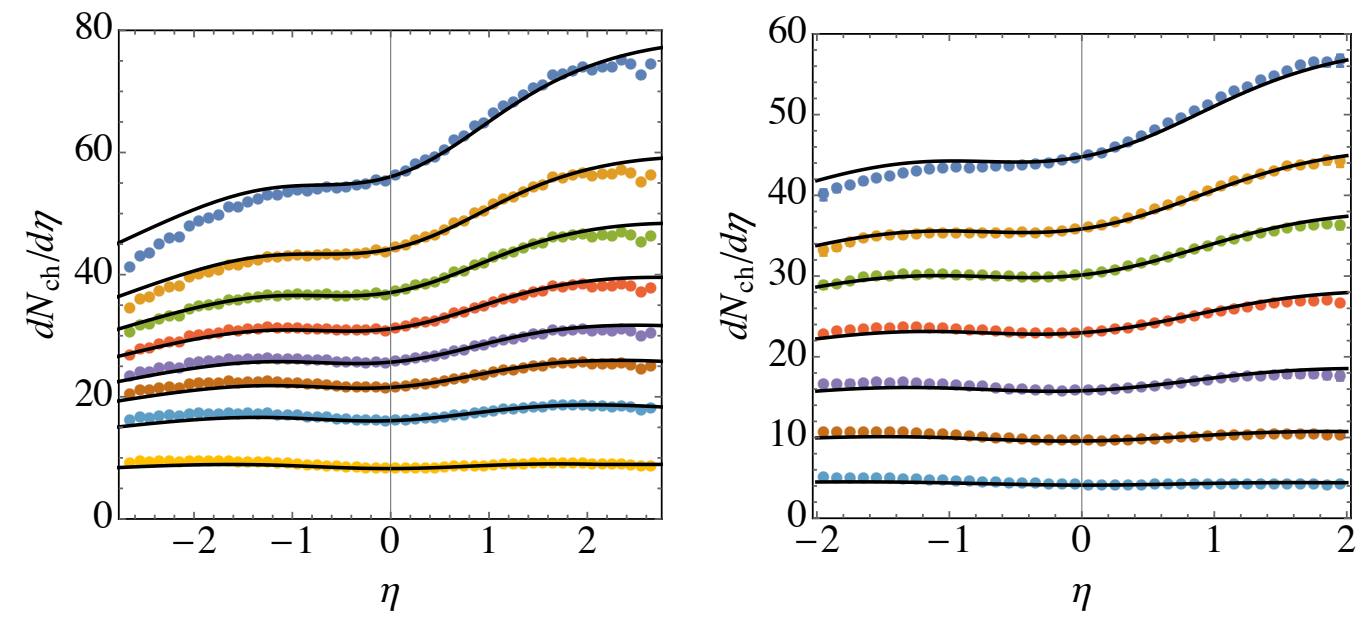

Figure 6: Multiplicity dependence on pseudo-rapidity $\eta$ for the fluctuating case with $\sigma=$ 1.55. Left plot corresponds to ATLAS whereas the right one to ALICE. Different curves correspond to the centrality classes defined in Tables 1 and 2.

Nucleon model. In both cases the shape of charge particle rapidity distribution is determined by the participant number $N_{\text {part }}$, and in both cases the simplest models of such a dependence fail to describe the data.

The idea that cross sections might fluctuate as an explanation for various phenomena in heavy ion collisions is not new. This was used in the Glauber-Gribov implementation to compute the number of participants in the ATLAS results described above. Motivation was found for the use of hydrodynamics in high multiplicity $\mathrm{p} A$ events at the LHC [28]. There have also been suggestions that this might be required from attempts to describe the ridge in $\mathrm{p} A$ collisions [29]. Furthermore fluctuations have been implemented phenomenologically in a Monte Carlo study of $\mathrm{p} A$ collisions done in Ref. [30] where the authors consider multiplicity variations for fixed $N_{\text {part }}$ given by the negative binomial distribution (NBD) that is obtained in the glasma model $[31,32]$. There our formula (14) corresponds to the average number of particles entering NBD. It is, however, difficult to assess the energy dependence of these fluctuations as it stems from the energy dependence of the NBD itself and of the impact parameter profile that selects given $N_{\text {part }}$ (that in a sense would correspond to the fluctuations of the nucleus saturation scale).

On the other hand, the arguments for a fluctuating saturation momentum have been primarily theoretical [16]-[25]. Interest in such fluctuations diminished somewhat when no evidence of diffusive scaling has been found 

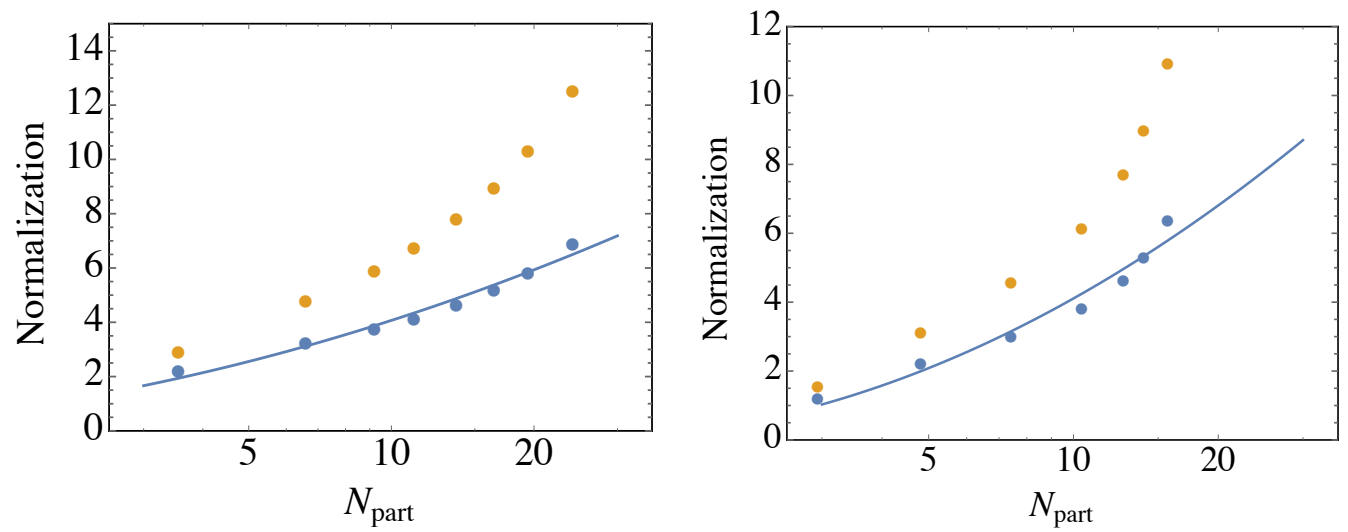

Figure 7: Normalization of multiplicity distributions shown in Fig 5 as functions of $N_{\text {part }}$. Left panel is for ATLAS and right panel for ALICE. Upper (orange) points correspond to the non-fluctuating case, whereas lower (blue) points correspond to fluctuations with $\sigma=1.55$. Parametrization of Eq. (24) is shown as a solid (blue) line.

in DIS [20, 22] and after it was argued that the evolution of the strength of such fluctuations was very weak with varying rapidity. However, if the fluctuations are largely rapidity independent - as it is the case of the present paper - geometrical scaling variable $\tau$ is not modified and there is no contradiction between our results and the absence of diffusive scaling in DIS. Another point is the energy dependence of such fluctuations. This needs to be tested in comparative studies at both RHIC and LHC energies.

Our finding that the fluctuations can account for the data must be taken with much caution. It is simply a first attempt and the number extracted for the width of the fluctuations of the logarithm is rather large, and exceeds by a factor of 1.5 the one of recent analysis of Ref. [33]. This may be because in our simple analysis, we do not consider fluctuations of the saturation momentum of the nucleus. It is not obvious how to do this, since the fluctuations would be local over some transverse scale size of order the inverse saturation momentum. Similarly, we expect there to be local structure in the proton since the size scale of the saturation momentum is small compared to the proton size. The issue of a local fluctuating saturation momentum was addressed by Iancu and McLerran [34] within the context of conformal field theory, but there is as yet no practical implementation of such ideas useful for understanding experimental data. Furthermore, in our simple analysis we have neglected other sources of fluctuations that have been taken into account in Ref. [33]. Finally, since we do not include any dumping for large $y$ 's 
(as has been done e.g. in Ref. [26] where large $x$ suppression for gluon densities has been included), our calculation is most reliable in the mid rapidity region. Evidently a more realistic model must contain such effects.

If we accept the idea of a fluctuating saturation momenta as a working hypothesis, there are a variety of tests one can imagine. Fluctuations in the multiplicity for very high multiplicity predicted in the IP-glasma model will be modified by such effects $[35,33]$. The correlation between multiplicity and jet production will also be changed, since higher saturation momenta in the proton means more gluons and therefore greater probability of producing a jet.

The original idea of studying multiplicity fluctuations in $\mathrm{p} A$ collisions was that they would allow probes of higher density regions inside the nucleus. The results we present in this paper suggest that the gluon content of the proton also itself changes when the multiplicity of final state particles varies.

\section{Acknowledgements}

We would like to thank A. Bialas, A. Bzdak, E. Iancu, D. Kharzeev, E. Levin, P. Tribedy and R. Venugopalan for discussion and remarks. The authors are supported under Department of Energy contract number Contract No. DE-SC0012704. Research of MP has been supported by the Polish NCN grant 2014/13/B/ST2/02486.

\section{References}

[1] A. Bzdak and V. Skokov, Phys. Rev. Lett. 111, 182301 (2013) [arXiv:1307.6168 [hep-ph]].

[2] L. D. McLerran, and R. Venugopalan, Phys. Rev. D49 (1994) 2233 , Phys. Rev. D49 (1994) 3352 and Phys. Rev. D50 (1994) 2225.

[3] A. Bialas, M. Bleszynski and W. Czyz, Nucl. Phys. B 111, 461 (1976).

[4] A. Bialas and W. Czyz, Acta Phys. Polon. B 36, 905 (2005) [hep$\mathrm{ph} / 0410265]$.

[5] Y. V. Kovchegov and A. H. Mueller, Nucl. Phys. B 529, 451 (1998) [hep-ph/9802440]. 
[6] A. Dumitru and L. D. McLerran, Nucl. Phys. A 700, 492 (2002) [hep$\mathrm{ph} / 0105268]$.

[7] [ATLAS Collaboration], ATLAS-CONF-2013-096 and ATLAS-CONF2013-117, http://cds.cern.ch/record/1599773, talk at the 15th conference on Elastic and Diffractive Scattering, Saariselka, Lapland, Finland, 9 - 13 Sep 2013;

B. Zabinski [ATLAS Collaboration], Acta Phys. Polon. B 45 (2014) 7, 1653 ;

R. R. Debbe [ATLAS Collaboration], Nucl. Phys. A 931 (2014) 872;

E. Shulga [ATLAS Collaboration], Nucl. Phys. A 926 (2014) 64;

A. Romaniouk [ATLAS Collaboration], ATL-PHYS-PROC-2014-002, http://cds.cern.ch/record/1640993.

[8] J. Adam et al. [ALICE Collaboration], arXiv:1412.6828 [nucl-ex].

[9] V. Guzey and M. Strikman, Phys. Lett. B 633, 245 (2006) [Phys. Lett. B 663, 456 (2008)] [hep-ph/0505088].

[10] M. Alvioli and M. Strikman, Phys. Lett. B 722, 347 (2013) [arXiv:1301.0728 [hep-ph]].

[11] J. L. Albacete et al., Int. J. Mod. Phys. E 22, 1330007 (2013) doi:10.1142/S0218301313300075 [arXiv:1301.3395 [hep-ph]].

[12] A. H. Rezaeian, Phys. Lett. B $718 \quad$ (2013) 1058 doi:10.1016/j.physletb.2012.11.066 [arXiv:1210.2385 [hep-ph]], and Phys. Rev. D 85 (2012) 014028 doi:10.1103/PhysRevD.85.014028 [arXiv:1111.2312 [hep-ph]].

[13] B. Schenke, S. Schlichting and R. Venugopalan, Phys. Lett. B 747 (2015) 76 doi:10.1016/j.physletb.2015.05.051 [arXiv:1502.01331 [hep-ph]].

[14] K. Dusling and R. Venugopalan, Nucl. Phys. A 931 (2014) 283. doi:10.1016/j.nuclphysa.2014.09.024

[15] B. Schenke, P. Tribedy and R. Venugopalan, Phys. Rev. C 89 (2014) no.2, 024901 doi:10.1103/PhysRevC.89.024901 [arXiv:1311.3636 [hep$\mathrm{ph}]$. 
[16] S. Munier and R. B. Peschanski, Phys. Rev. D 69, 034008 (2004) [hep$\mathrm{ph} / 0310357]$.

[17] S. Munier and R. B. Peschanski, Phys. Rev. Lett. 91, 232001 (2003) [hep-ph/0309177].

[18] J. Jalilian-Marian, A. Kovner, A. Leonidov, and H. Weigert, Nucl. Phys. B504, 415 (1997), and Phys. Rev. D59, 014014 (1998);

E. Iancu, A. Leonidov, and L. D. McLerran, Nucl. Phys. A692, 583 (2001);

E. Ferreiro, E. Iancu, A. Leonidov, and L. D. McLerran, Nucl. Phys. A703, 489 (2002).

[19] I. Balitsky, Nucl. Phys. B463, 99 (1996);

Y. V. Kovchegov, Phys. Rev. D60, 034008 (1999), and Phys. Rev. D61, 074018 (2000).

[20] F. Gelis, R. B. Peschanski, G. Soyez and L. Schoeffel, Phys. Lett. B 647, 376 (2007) [hep-ph/0610435].

[21] G. Beuf, R. Peschanski, C. Royon and D. Salek, Phys. Rev. D 78, 074004 (2008) [arXiv:0803.2186 [hep-ph]].

[22] M. Praszalowicz and T. Stebel, JHEP 1304, 169 (2013) [arXiv:1302.4227 [hep-ph]].

[23] E. Iancu, A. H. Mueller and S. Munier, Phys. Lett. B 606, 342 (2005) [hep-ph/0410018].

[24] Y. Hatta, E. Iancu, C. Marquet, G. Soyez and D. N. Triantafyllopoulos, Nucl. Phys. A 773, 95 (2006) [hep-ph/0601150].

[25] A. Dumitru, E. Iancu, L. Portugal, G. Soyez and D. N. Triantafyllopoulos, JHEP 0708, 062 (2007) [arXiv:0706.2540 [hep-ph]].

[26] D. Kharzeev, E. Levin and M. Nardi, Nucl. Phys. A 747, 609 (2005) [hep-ph/0408050].

[27] M. Praszalowicz and T. Stebel, JHEP 1303, 090 (2013) [arXiv:1211.5305 [hep-ph]].

[28] C. Coleman-Smith and B. Müller, Annals Phys. 352 (2015) 59. 
[29] K. Dusling and R. Venugopalan, Phys. Rev. D 87 (2013) 9, 094034 [arXiv:1302.7018 [hep-ph]].

[30] A. Dumitru, D. E. Kharzeev, E. M. Levin and Y. Nara, Phys. Rev. C 85, 044920 (2012) [arXiv:1111.3031 [hep-ph]].

[31] F. Gelis, T. Lappi and L. McLerran, Nucl. Phys. A 828, 149 (2009) [arXiv:0905.3234 [hep-ph]].

[32] P. Tribedy and R. Venugopalan, Nucl. Phys. A 850, 136 (2011) [Nucl. Phys. A 859, 185 (2011)] [arXiv:1011.1895 [hep-ph]].

[33] L. McLerran and P. Tribedy, Nucl. Phys. A 945, 216 (2016) doi:10.1016/j.nuclphysa.2015.10.008 [arXiv:1508.03292 [hep-ph]].

[34] E. Iancu and L. McLerran, Nucl. Phys. A 793 (2007) 96 [hepph/0701276 [hep-ph]].

[35] B. Schenke, P. Tribedy and R. Venugopalan, Phys. Rev. C 86 (2012) 034908 [arXiv:1206.6805 [hep-ph]]. 